\title{
A Calibration Method of USBL Installation Error Based on Attitude Determination
}

\author{
Yongyun Zhu, Tao Zhang, Shouquan Xu, Hyo-Sang Shin, Peijuan Li, Bonan Jin, Liang Zhang, Chengcheng Weng, \\ Yao Li
}

\begin{abstract}
The Ultra-short baseline (USBL) positioning system has important application in the positioning of underwater vehicles. The installation error angle of the USBL positioning system has an important influence on the positioning accuracy of USBL system. The traditional calibration methods have limited estimation accuracy for installation error angles and have high route requirements. To solve the above problems, a calibration method of installation error angle based on attitude determination is proposed in this paper. When strapdown inertial navigation system (SINS) and USBL are fixed together in the application process, the installation error angle of USBL is fixed and unchanged. Then the calibration of installation error angle can be accomplished with the idea of attitude determination. The vector observation model based on the installation error angle matrix is established first. Observation vectors are obtained by the relative position of transponders in the USBL coordinate frame. The reference vector is calculated by position of transponder, position and attitude of SINS and lever arm between SINS and USBL. By constructing the observation vectors and the reference vectors, the proposed method can calibrate the installation error angle of SINS and USBL in real time. The advantages of the proposed method are that it has no specific requirements for the calibration route and can calibrate the installation error angle in real time
\end{abstract}

Copyright (c) 2015 IEEE. Personal use of this material is permitted. However, permission to use this material for any other purposes must be obtained from the IEEE by sending a request to pubs-permissions@ieee.org.

Manuscript received xxxx, xxxx; revised xxxx, xxxx; accepted xxxx, xxxx. This work was supported in part by the Inertial Technology Key Lab Fund under Grant 614250607011709 ,in part by the Fundamental Research Funds for the Central Universities under Grant 2242019K40041 and 2242020k1G009, Key Laboratory Fund for Underwater Information and Control under Grant 614221805051809, and in part by the Jiangsu Key Laboratory Fund for Green Ship Technology under Grant 2019Z01, Remaining funds cultivation project of National Natural Science Foundation of Southeast University under Grant 9S20172204, and in part by the Postgraduate Research \& Practice Innovation Program of Jiangsu Province KYCX19_0088, in part by the Natural Science Foundation of Jiangsu Province, China under Grant BK20190344, in part by the Chinese Defense Leading Program of Science and Technology under Grant 1816300TS00402001.

(Corresponding author: Tao Zhang.) Y. Y. Zhu, T. Zhang, B. N. Jin, L. Zhang, C. C. Weng and Y. Li are with School of Instrument Science \& Engineering, Southeast University, Nanjing 210096, China and the Key Laboratory of Micro-Inertial Instrument and Advanced Navigation Technology, Ministry of Education, Southeast University, Nanjing 210096, China (e-mail: zhyy@seu.edu.cn; zhangtao22@seu.edu.cn; jinbonan@seu.edu.cn; zhangliang418@seu.edu.cn; wengchengcheng@seu.edu.cn; liyao@seu.edu.cn).

S. Q. Xu is with Jiangsu Automation Research Institute, Lianyungang 222061, China (e-mail: xushouquan120@126.com).

H. S. Shin is with the Institute of Aerospace Sciences, SATM, Cranfield University, MK43 0AL Cranfield, U.K., E-mail: (h.shin@cranfield.ac.uk).

P. J. Li is with Nanjing Institute of Technology, Nanjing 211167, China (e-mail: 1pj4444@126.com) with high accuracy. In order to verify the performance of the proposed algorithm, simulation experiment and field experiment are carried out in this paper. The results of simulation experiment and field experiment show that the proposed method can give the estimated installation error angle of USBL in real time, and the estimated result is the best among several methods. The proposed method can not only achieve the calibration of the installation error angle in circular trajectory, but also in straight trajectory.

Index Terms - Ultra-short baseline (USBL); installation error angle; attitude determination; observation vectors; Real-time calibration;

\section{INTRODUCTION}

A utonomous underwater vehicles (AUV) is an indispensable carrier for human to investigate and study marine resources [1-2]. Underwater positioning technology is one of the key technologies for underwater vehicles to carry out underwater operations [3-5]. The medium environment of the ocean determines that sound is more suitable as a propagation carrier for underwater positioning technology than light and electromagnetic waves [6-7]. According to the different baseline length of receiving array, available underwater acoustic positioning system usually includes three kinds: long baseline (LBL) system, short baseline (SBL) system and ultra-short baseline (USBL) system [8-9]. USBL system has the advantages of simple structure, easy operation and small size, which makes it play an increasingly important role in the field of marine resources investigation and scientific research [9-10].

USBL system can obtain the relative position between acoustic array and transponder. It is necessary to convert the relative position to global position of AUV in navigation coordinate frame by attitude sensor. The attitude of AUV is usually provided by strapdown inertial navigation system (SINS). In order to avoid the accumulation of position errors, SINS often needs other positioning systems to assist in underwater navigation. Therefore, the SINS/USBL integrated navigation system has become the main development direction of AUV underwater positioning technologies [11-13]. In 2006, Morgado first proposed a tightly-coupled USBL/INS integration strategy that uses the USBL slant range value to complete the high-precision underwater positioning [11]. In order to verify the effectiveness of the method, Morgado carried out simulation experiments in 2006 and experimental trials at sea in Portugal in 2013 [12].

The main factors affecting the positioning accuracy of the 
USBL system are the system error, the measurement error caused by ocean environmental parameters and the installation error of transceiver array [14-15]. Installation error angle is caused by the inconsistency between the acoustic hydrophone array frame and the inertial navigation body frame. Acoustic array installation error is the main error source of positioning system, and it must be calibrated accurately before practical use. Taking the USBL system with positioning accuracy of $0.5 \%$ slant range as an example, an installation error of 1 degree in heading angle will cause a positioning error of at least $1.7 \%$ slant range [16]. Therefore, in order to improve the positioning accuracy of USBL system, the problem of installation error needs to be solved first.

Although the calibration of installation errors plays an essential feature in improving the positioning accuracy of USBL system, there are few relevant published literatures. Zheng Cuie of Harbin Engineering University proposed a calibration method using the least squares to estimate the installation error of USBL system, and carried out the experiment at sea in 2007 [16]. However, when the method solves the installation error angle by the least square method, the matrix needs to be inversed, resulting in singular values in the algorithm. Then, several other scholars also published several articles on the installation error calibration, but these methods are all based on the least squares theory to complete the installation error angle estimation [17-19]. Chen Hsin-Hung designed an iterative calibration method to estimate the installation error angle by analyzing the influence of three attitudes in the installation error angle on USBL positioning system respectively [9]. But Cheng Xinhong's method has high requirements for the calibration route. The calibration route is difficult to achieve in actual experiments. Tong Jinwu designed a dual transponder-based calibration method for USBL system by referring to Cheng Hsin-Hung's method [20]. But Tong's method requires precise knowledge of the depth difference between the two transponders, which is difficult to meet in the experimental environment. Sun Dajun proposed a method to solve the installation error angle by matrix decomposition [14]. However, Sun Dajun's method is similar to Cheng Xinhong's, both of which are solved by dividing the three angles of installation error and using iterative estimator. Through the analysis of the existing calibration papers, it can be found that the classical methods use least squares to estimate the installation error angle of USBL, while the state-of-the-art methods utilize matrix decomposition to complete the calibration of the installation error angle. The disadvantage of least square method lies in the existence of singular values, while the calibration route of matrix decomposition method is too complicated. Therefore, in order to improve the performance of calibration, it is inevitable to develop a simple-operated and accurate calibration method.

In practical application, the SINS and the acoustic hydrophone array of the USBL are fixed on the ship. The installation error between the b-frame of SINS and the a-frame of USBL is a constant value, so that the calibration of installation error can be solved by the method of attitude determination. The attitude determination was first proposed to solve the attitude of the spacecraft. The task of attitude determination is that of determining the orientation of the spacecraft relative to some reference frame [21]. The application of attitude determination can be extended to solve the transformation matrix between two coordinate frames. There are many methods for determining the attitude. One important branch is to solve the attitude determination process by solving the corresponding attitude quaternion [21]. By constructing the appropriate observation vectors and reference vectors, the calibration of the installation error can be done with the idea of attitude determination. In SINS, the nine-component attitude matrix has only three independent parameters because of the orthogonality constraint $\boldsymbol{A A}^{T}=\boldsymbol{I}$ [22]. Every constructed three-dimensional vector contains more than two independent scalar pieces of attitude information. Owing to the norm constraint, each of the unit vectors contains only two independent scalar pieces of attitude information [23]. Two non collinear three-dimensional vectors contain more than four independent variables. Consequently, as long as two non collinear vectors are constructed, the attitude matrix can be sufficiently (unnecessarily) solved. In addition, it should be noted that since the a-frame and b-frame do not coincide, the vectors in a-frame and b-frame will not be collinear in the actual experiment. Therefore, how to construct reference vector and observation vector is the key step to complete the real time calibration for USBL installation error angle. In this paper, the observation vector and reference vector are obtained by USBL positioning and SINS positioning respectively, and the optimization-based quaternion method is used to complete the calibration of the installation error angle. On the other hand, the attitude result of SINS is needed in the calibration method. In order to make the calibration result more accurate, the attitude result of SINS/GPS integrated navigation is used in the calibration method. The calibration method proposed in this paper is convenient for operation, and can be used for real-time calibration of installation error.

The rest of the article is structured as follows. The principle of USBL positioning and the definition of installation error angle are first introduced in Section 2. In the Section 3, the principle of SINS/GPS integrated navigation is described. SINS/GPS integrated navigation system is used in this paper to get a high-precision attitude and position results, which guarantee the calibration accuracy of USBL installation error angle. The principle of the calibration method proposed in this paper is presented in Section 4. The vector construction of the calibration method is given in Section 4.1, while the attitude determination of the calibration method is described in Section 4.2. In the Section 5, the simulation experiment of calibration method is carried out, and the analysis based on numerical simulation results is presented. The experimental evaluation and validation of the proposed technique is reported in Section 6 . Finally, the conclusion and comments of this paper are given in Section 7. 


\section{USBL POSITION AND INSTALLATION ERROR}

\section{A. Definition of the coordinate frame}

In order to introduce the principle of ultra-short baseline positioning and the calibration method of installation error more clearly and conveniently, this paper first introduces the definitions of several coordinate systems used in this paper.

(1) $b$-frame: The body frame for SINS (Right-Forth-Up, $\mathrm{RFU})$. The origin of the $\mathrm{b}$-frame is located at the center of the IMU.

(2) $n$-frame: The local level navigation frame (East-North-Up, ENU).

(3) $a$-frame: The acoustic hydrophone array frame for USBL (Right-Forth-Up, RFU). The origin of the a-frame is located at the center of the hydrophone array.

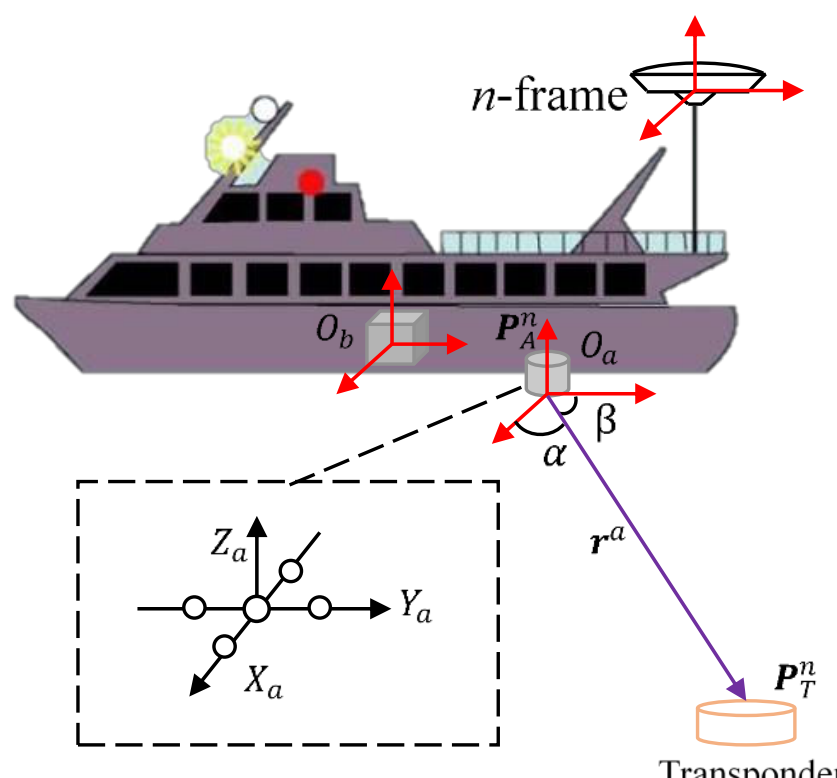

Transponder

Fig. 1. Definitions of coordinate frames and installation error angle.

As shown in Fig 1, since the IMU and the acoustic hydrophone array are not installed together, so there is a fixed displacement between the origin of the $b$-frame $O_{b}$ and the origin of the $a$-frame $O_{a}$. Besides, there are installation angle errors between the three axes of the $b$-frame and those of the $a$-frame. The displacement between the origins of b-frame $O_{b}$ and a-frame $O_{a}$ can be measured, and the residual errors after measurement can be neglected compared with the positioning errors of USBL. (Note: Assume that the positioning accuracy of USBL system is $1 \% \mathrm{R}$, where $\mathrm{R}$ is the slant range. Then, the residual error of lever measurement should be within $0.1 \%$ of the slant range in the experiment. For example, assume that the slant range of USBL system in the experiment is $100 \mathrm{~m}$. Therefore, the measurement error of lever should be within $10 \mathrm{~cm}$, which can ensure that the influence of the residual lever error on the positioning accuracy of the USBL system can be ignored.) Even if the USBL system has a small installation angle error between the b-frame and a-frame, it will have a great impact on the USBL positioning error. Since the installation angle error cannot be accurately measured manually, the installation angle error can only be obtained by a specific calibration method. The three installation error angles between the b-frame and a-frame are expressed as $\left[\begin{array}{lll}\theta & \gamma & \phi\end{array}\right]$ respectively. Then the attitude matrix between the b-frame and a-frame can be described as:

$$
\begin{aligned}
\boldsymbol{C}_{a}^{b}=\left[\begin{array}{ccc}
\cos \gamma & 0 & -\sin \gamma \\
0 & 1 & 0 \\
\sin \gamma & 0 & \cos \gamma
\end{array}\right]\left[\begin{array}{ccc}
1 & 0 & 0 \\
0 & \cos \theta & \sin \theta \\
0 & -\sin \theta & \cos \theta
\end{array}\right] \\
\\
{\left[\begin{array}{ccc}
\cos \phi & -\sin \phi & 0 \\
\sin \phi & \cos \phi & 0 \\
0 & 0 & 1
\end{array}\right] }
\end{aligned}
$$

\section{B. The principle of the USBL positioning}

The USBL positioning system usually consists of two parts, one is the small and compact acoustic hydrophone array and transducer installed on the bottom of the ship, and the other is the transponder installed on the seabed. As shown in Fig 2(b), the acoustic hydrophone array consists of four or more regularly installed hydrophones. In order to be consistent with the USBL equipment used in later filed test, this paper assumes that the acoustic hydrophone array contains four hydrophones. The four hydrophones arranged in a square are named 1, 2, 3 and 4 respectively. The transducer $\mathrm{O}$ is located in the center of four hydrophones. The origin of a-frame is set as the position of transducer. The direction from transducer $\mathrm{O}$ to hydrophone 4 is defined as the positive $\mathrm{X}$-axis direction of a-frame. The direction from transducer $\mathrm{O}$ to hydrophone 3 is defined as the positive $\mathrm{Y}$-axis direction of a-frame. The $\mathrm{Z}$-axis of a-frame is upward and perpendicular to the $\mathrm{X}$-axis and $\mathrm{Y}$-axis respectively. As showed in Fig 2(a), the transducer installed on the vehicle first sends an acoustic wave to the transponder placed at a known position. The transponder responds to the receivers on the USBL array with a reply when receiving the signal. Fig 2(b) presents the definition of directions and slant range in USBL system. The slant range $R$ between the transponder and the acoustic array can be obtained by measuring the propagation time of the acoustic signal and multiplying the velocity of the acoustic wave in the water. The underwater sound speed varies with the change of physical properties of water such as temperature and salinity. The sound velocity can be considered as a constant value when the vessel is operating in the same underwater layer as the transponder. The straight line between the transponder and the transducer is recorded as the line of slant range. The directions $\left[\begin{array}{ll}\alpha & \beta\end{array}\right]$ of the USBL system can be obtained by measuring the angle between the line of slant range and the $\mathrm{x}$-axis and the $\mathrm{y}$-axis of the a-frame respectively. 


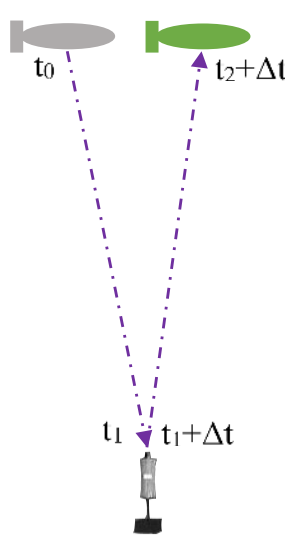

(a)

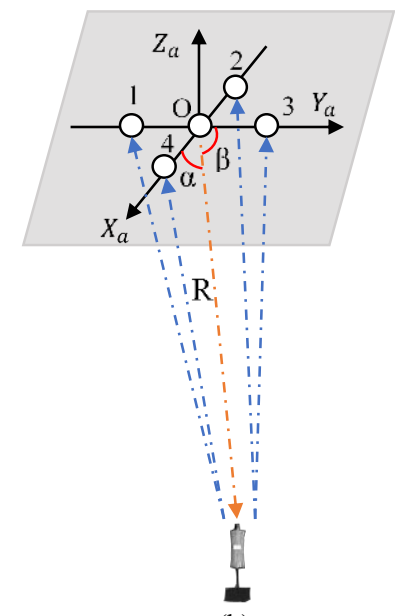

(b)
Fig. 2. (a) Travel path of the acoustic waves in a Location Period of USBL System; (b) Definition of directions and slant range in USBL system.

The position of the transponder in the n-frame $\boldsymbol{P}_{T}^{n}$ has been obtained by LBL positioning system at the time of installation. Then the relative position of the transponder in the a-frame can be obtained by calculating the slant distance $\mathrm{R}$ and directions $\left[\begin{array}{ll}\alpha & \beta\end{array}\right]$.

$$
\boldsymbol{r}^{a}=\left[\begin{array}{l}
x \\
y \\
z
\end{array}\right]=\left[\begin{array}{c}
R \cos \alpha \\
R \cos \beta \\
-R \sqrt{1-(\cos \alpha)^{2}-(\cos \beta)^{2}}
\end{array}\right]
$$

(2) gives the calculation process of solving $\boldsymbol{r}^{a}$ by USBL system. On the other hand, the following equivalence relation is established for $\boldsymbol{r}^{a}$.

$$
\boldsymbol{r}^{a}=\boldsymbol{C}_{b}^{a} \boldsymbol{C}_{n}^{b} \boldsymbol{r}^{n}=\boldsymbol{C}_{b}^{a} \boldsymbol{C}_{n}^{b}\left(\boldsymbol{P}_{T}^{n}-\boldsymbol{P}_{A}^{n}\right)
$$

Where the matrix $\boldsymbol{C}_{b}^{a}=\left(\boldsymbol{C}_{a}^{b}\right)^{T}$ is the installation error matrix to be solved. The matrix $\boldsymbol{C}_{n}^{b}$ represents the attitude matrix between the $b$-frame and the $n$-frame, which can be obtained by the SINS. $\boldsymbol{r}^{n}$ is the relative position of the transponder in the n-frame. $\boldsymbol{P}_{T}^{n}$, obtained by LBL positioning system, is the position of the transponder in the n-frame. $\boldsymbol{P}_{A}^{n}$ is the position of the hydrophone in the $n$-frame and the final positioning results of USBL position system.

According to (3), position $\boldsymbol{P}_{A}^{n}$ can be calculated as follows.

$$
\boldsymbol{P}_{A}^{n}=\boldsymbol{P}_{T}^{n}-\boldsymbol{r}^{n}=\boldsymbol{P}_{T}^{n}-\boldsymbol{C}_{b}^{n} \boldsymbol{C}_{a}^{b} \boldsymbol{r}^{a}
$$

Where the matrix $\boldsymbol{C}_{b}^{n}$ is the transposed matrix of matrix $\boldsymbol{C}_{n}^{b}$. The following information can be got by analyzing (4). On the one hand, only after the installation error angle of USBL system is solved, the position result of USBL system can be obtained. The accuracy of installation error matrix $\boldsymbol{C}_{a}^{b}$ directly affects the performance of USBL positioning system. As for how to use attitude determination method to solve the installation error angle, it will be summarized in Section 4. On the other hand, the positioning accuracy of the USBL system is also subject to the accuracy of attitude matrix $\boldsymbol{C}_{b}^{n}$. In order to make the attitude result more accurate, we use the attitude result of the SINS/GPS integrated navigation system instead of directly using the SINS attitude result. The specific idea of using SINS/GPS integrated navigation to solve more accurate attitude angle will be introduced in Section 3.

\section{SINS/GPS INTEGRATION NAVIGATION}

Since the attitude matrix $\boldsymbol{C}_{b}^{n}$ provided by SINS is needed in USBL positioning system and calibration method, this section focuses on the principle of SINS providing accurate attitude. Due to the influence of inertial sensors error, SINS attitude error will accumulate gradually, which is obviously unfavorable for USBL positioning. If there is GPS information to assist SINS, GPS position information can be used as measurement information to correct SINS attitude result and suppress attitude error divergence. In order to make the calibration result more accurate, the SINS/GPS integrated navigation system is used to provide the attitude matrix $\boldsymbol{C}_{b}^{n}$ required by the calibration method. The real-time kinematic (RTK) positioning of GPS with ambiguity integer solution is utilized for SINS/GPS integration navigation to complete the calibration of SINS attitude.

According to the error characteristics of the SINS, the position errors, velocity errors, attitude errors, gyroscope drift and accelerometer bias are selected as the state vectors.

$$
\mathrm{X}=\left[\begin{array}{cccccccc}
\phi_{E} & \phi_{N} & \phi_{U} & \delta V_{E} & \delta V_{N} & \delta V_{U} & \delta L \\
\delta \lambda & \delta h & \varepsilon_{x} & \varepsilon_{y} & \varepsilon_{z} & \nabla_{x} & \nabla_{y} & \nabla_{z}
\end{array}\right]
$$

where $\left[\begin{array}{lll}\phi_{E} & \phi_{N} & \phi_{U}\end{array}\right]$ is the attitude error vector of SINS. $\left[\begin{array}{lll}\delta V_{E} & \delta V_{N} & \delta V_{U}\end{array}\right]$ is the velocity error vector of SINS. $\left[\begin{array}{lll}\delta L & \delta \lambda & \delta h\end{array}\right]$ is the position error vector of SINS. $\left[\begin{array}{lll}\varepsilon_{x} & \varepsilon_{y} & \varepsilon_{z}\end{array}\right]$ is the constant error vector of gyroscopes. $\left[\begin{array}{lll}\nabla_{x} & \nabla_{y} & \nabla_{z}\end{array}\right]$ is the constant error vector of accelerometers.

The state equation of SINS is expressed as follow

$$
\dot{\mathbf{X}}(t)=\boldsymbol{F}(t) \boldsymbol{X}(t)+\boldsymbol{W}(t)
$$

where $\boldsymbol{X}(t)$ is the state vectors of the state equation. $\boldsymbol{F}(t)$ is the state transition matrix of the state equation. $\boldsymbol{W}(t)$ is the noise vector of SINS.

$$
\begin{aligned}
& \mathbf{F}(t)=\left[\begin{array}{ccccc}
\boldsymbol{F}_{11} & \boldsymbol{F}_{12} & \boldsymbol{F}_{13} & -\boldsymbol{C}_{b}^{n} & \mathbf{0}_{3 \times 3} \\
\boldsymbol{F}_{21} & \boldsymbol{F}_{22} & \boldsymbol{F}_{23} & \mathbf{0}_{3 \times 3} & \boldsymbol{C}_{b}^{n} \\
\mathbf{0}_{3 \times 3} & \boldsymbol{F}_{32} & \boldsymbol{F}_{33} & \mathbf{0}_{3 \times 3} & \mathbf{0}_{3 \times 3} \\
\mathbf{0}_{3 \times 3} & \mathbf{0}_{3 \times 3} & \mathbf{0}_{3 \times 3} & \boldsymbol{F}_{44} & \mathbf{0}_{3 \times 3} \\
\mathbf{0}_{3 \times 3} & \mathbf{0}_{3 \times 3} & \mathbf{0}_{3 \times 3} & \mathbf{0}_{3 \times 3} & \boldsymbol{F}_{55}
\end{array}\right] \\
& \text { Where } \boldsymbol{F}_{11}=\left[\begin{array}{ccc}
0 & \omega_{i n}^{n}(3) & -\omega_{i n}^{n}(2) \\
-\omega_{i n}^{n}(3) & 0 & \omega_{i n}^{n}(1) \\
\omega_{i n}^{n}(2) & -\omega_{i n}^{n}(1) & 0
\end{array}\right], \\
& \boldsymbol{F}_{12}=\left[\begin{array}{ccc}
0 & -1 / R_{M h} & 0 \\
1 / R_{N h} & 0 & 0 \\
\tan L / R_{N h} & 0 & 0
\end{array}\right], \\
& R_{M h}=R_{M}+h, R_{N h}=R_{N}+h,
\end{aligned}
$$




$$
\begin{aligned}
& \boldsymbol{F}_{13}=\left[\begin{array}{ccc}
0 & 0 & V_{N} / R_{M h}^{2} \\
-\omega_{i e} \sin L & 0 & -V_{E} / R_{N h}^{2} \\
\omega_{i e} \cos L+V_{E} \sec ^{2} L / R_{N h} & 0 & -V_{E} \tan L / R_{N h}^{2}
\end{array}\right] \text {, } \\
& \boldsymbol{F}_{21}=\left[\begin{array}{ccc}
0 & -f_{U} & f_{N} \\
f_{U} & 0 & -f_{E} \\
-f_{N} & f_{E} & 0
\end{array}\right], \\
& \boldsymbol{F}_{22}=\left(\boldsymbol{V}^{n} \times\right) \boldsymbol{F}_{12}-(\boldsymbol{a} \times) \text {, } \\
& \boldsymbol{a}=\left[\begin{array}{lll}
\frac{-V_{N}}{R_{M h}} & 2 \omega_{i e} \cos L+\frac{V_{E}}{R_{N h}} & 2 \omega_{i e} \sin L+\frac{V_{E} \tan L}{R_{N h}}
\end{array}\right]^{T}, \\
& \boldsymbol{F}_{32}=\left[\begin{array}{ccc}
0 & 1 / R_{M h} & 0 \\
\sec L / R_{N h} & 0 & 0 \\
0 & 0 & 1
\end{array}\right] \text {, } \\
& \boldsymbol{F}_{33}=\left[\begin{array}{ccc}
0 & 0 & -V_{N} / R_{M h}^{2} \\
V_{E} \tan L \sec L / R_{N h} & 0 & -V_{E} \sec L / R_{N h}^{2} \\
0 & 0 & 0
\end{array}\right], \\
& \boldsymbol{F}_{44}=\left[\begin{array}{ccc}
-1 / \tau_{g x} & 0 & 0 \\
0 & -1 / \tau_{g y} & 0 \\
0 & 0 & -1 / \tau_{g z}
\end{array}\right] \text {, } \\
& \boldsymbol{F}_{55}=\left[\begin{array}{ccc}
-1 / \tau_{a x} & 0 & 0 \\
0 & -1 / \tau_{a y} & 0 \\
0 & 0 & -1 / \tau_{a z}
\end{array}\right] \text {. }
\end{aligned}
$$

The real-time kinematic (RTK) positioning of GPS with ambiguity integer solution can be used to provide precise position for SINS in USBL calibration. The position difference between SINS and RTK can be used as the observation of SINS/GPS loose combination. The measurement equation of the SINS/GPS integrated navigation is as the following equation.

$$
\mathbf{Z}(t)=\boldsymbol{P}_{S I N S}-\boldsymbol{P}_{G P S}=\boldsymbol{H}(t) \boldsymbol{X}(t)+\boldsymbol{V}(t)
$$

where $\mathbf{Z}(t)$ is the observation vector of the measurement equation. $\boldsymbol{H}(t)$ is the observation matrix of the measurement equation. $\boldsymbol{V}(t)$ is the observation noise of the measurement equation.

$$
\boldsymbol{H}(t)=\left[\begin{array}{lllll}
\mathbf{0}_{3 \times 3} & \mathbf{0}_{3 \times 3} & \boldsymbol{I}_{3 \times 3} & \mathbf{0}_{3 \times 3} & \mathbf{0}_{3 \times 3}
\end{array}\right]
$$

First, the state equation and measurement equation are discretized.

$$
\left\{\begin{array}{c}
\boldsymbol{X}_{k}=\boldsymbol{\Phi}_{k, k-1} \boldsymbol{X}_{k-1}+\boldsymbol{W}_{k-1} \\
\boldsymbol{Z}_{k}=\boldsymbol{H}_{k} \boldsymbol{X}_{k}+\boldsymbol{V}_{k}
\end{array}\right.
$$

Where $\boldsymbol{X}_{k}$ and $\boldsymbol{X}_{k-1}$ are the state vectors at time $t_{k}$ and $t_{k-1}$ respectively. $\boldsymbol{\Phi}_{k, k-1}$ is the state transition matrix from time $t_{k-1}$ to $t_{k} \cdot \boldsymbol{W}_{k-1}$ is the system noise vector at time $t_{k-1} . \boldsymbol{Z}_{k}$ is the measurement vector at time $t_{k} . \boldsymbol{H}_{k}$ is the observation matrix at time $t_{k} . \boldsymbol{V}_{k}$ is the observation noise vector at time $t_{k}$.

The Kalman filter is summarized as follows

$$
\begin{gathered}
\boldsymbol{X}_{k / k-1}=\boldsymbol{\Phi}_{k, k-1} \boldsymbol{X}_{k-1} \\
\boldsymbol{X}_{k}=\boldsymbol{X}_{k / k-1}+\boldsymbol{K}_{k}\left(\boldsymbol{Z}_{k}-\boldsymbol{H}_{k} \boldsymbol{X}_{k / k-1}\right) \\
\boldsymbol{K}_{k}=\boldsymbol{P}_{k / k-1} \boldsymbol{H}_{k}^{T}\left(\boldsymbol{H}_{k} \boldsymbol{P}_{k / k-1} \boldsymbol{H}_{k}^{T}+\boldsymbol{R}_{k}\right)^{-1} \\
\boldsymbol{P}_{k / k-1}=\boldsymbol{\Phi}_{k, k-1} \boldsymbol{P}_{k-1} \boldsymbol{\Phi}_{k, k-1}^{T}+\boldsymbol{Q}_{k-1}
\end{gathered}
$$

$$
\boldsymbol{P}_{k}=\left(\boldsymbol{I}-\boldsymbol{K}_{k} \boldsymbol{H}_{k}\right) \boldsymbol{P}_{k / k-1}\left(\boldsymbol{I}-\boldsymbol{K}_{k} \boldsymbol{H}_{k}\right)^{T}+\boldsymbol{K}_{k} \boldsymbol{R}_{k} \boldsymbol{K}_{k}^{T}
$$

Because the calibration experiments are usually carried out on the lake or sea surface, this unoccupied regions are the best scene for GPS positioning. Therefore, the results of SINS/GPS integrated navigation are also of high accuracy and good stability. It should be noted that the SINS/GPS integrated navigation system can be solved in real time. Besides, the update frequency of SINS is $200 \mathrm{~Hz}$, while that of GPS is $1 \mathrm{~Hz}$. Therefore, after time update carried for 200 times, the measurement update can be carried out in the operation of integrated navigation algorithm.

\section{PRINCIPLE OF CALIBRATION METHOD}

In the application of SINS/USBL integrated navigation system, the IMU of SINS and the hydrophone array of USBL are usually fixed on the vehicle. The attitude matrix of installation errors between b-frame of SINS and a-frame of USBL is a constant value. Therefore, the calibration of the installation error angle can be solved by attitude determination method. The calibration method based on attitude determination needs to construct position vectors of transponder relative to the $b$-frame and $a$-frame. The schematic diagram of the calibration method is shown in the Fig 3.

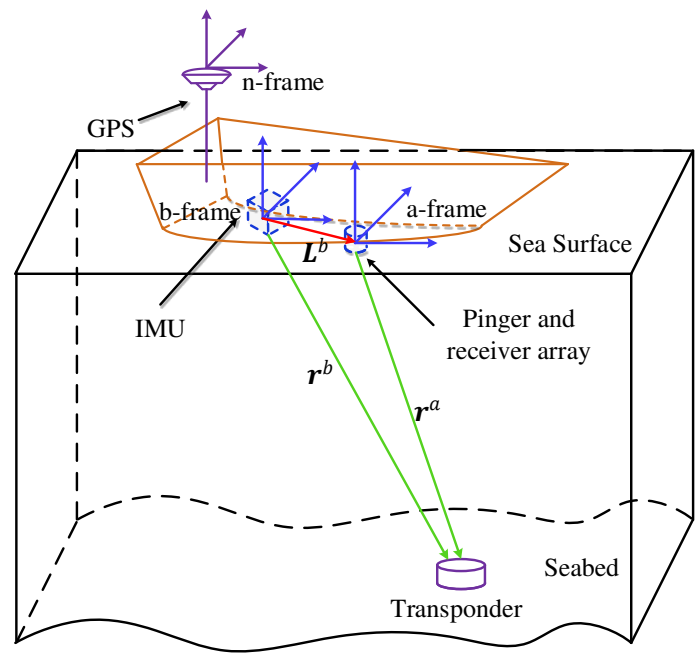

Fig. 3. The schematic diagram of proposed calibration method based on attitude determination.

\section{A. Vector construction}

As shown in the Fig $3, \boldsymbol{r}^{a}$, obtained by the USBL positioning system, represents the position vector of the transponder in the a-frame. The main factors affecting the accuracy of $\boldsymbol{r}^{a}$ include the measurement accuracy of slant range and directions in USBL system. The $\boldsymbol{r}^{b}$ in the Fig 3 represents the position vector of the transponder in the b-frame, which can be obtained by the following equation.

$$
\boldsymbol{r}^{b}=\boldsymbol{C}_{n}^{b}\left(\boldsymbol{P}_{T}^{n}-\boldsymbol{P}_{A}^{n}\right)
$$

Where $\boldsymbol{C}_{n}^{b}$ represents the attitude matrix between the b-frame and the n-frame. $\boldsymbol{P}_{A}^{n}$ represents the position of SINS in n-frame. Both the attitude matrix $\boldsymbol{C}_{n}^{b}$ and position $\boldsymbol{P}_{A}^{n}$ can be obtained 
from the results of the SINS/GPS integrated navigation system. $\boldsymbol{P}_{T}^{n}$, obtained by the LBL position system, represents the position of the transponder in $\mathrm{n}$-frame. Because the positioning accuracy of GPS with ambiguity integer solution is obviously better than USBL system, the attitude and position results of SINS/GPS integrated navigation system have enough high accuracy, which provides a guarantee for the calibration of installation error of USBL system. The position accuracy of LBL positioning system is much higher than that of USBL positioning system. Therefore, it can be considered that $\boldsymbol{r}^{b}$ has high positioning accuracy.

The vector observations-based measurement model for attitude matrix of installation error $\boldsymbol{C}_{b}^{a}$ is established as

$$
\boldsymbol{C}_{b}^{a}\left(\boldsymbol{r}^{b}-\boldsymbol{L}^{b}\right)=\boldsymbol{r}^{a}
$$

Where $\boldsymbol{L}^{b}$ denotes the projection in b-frame of the distance between the origin of b-frame $O_{b}$ and the origin of a-frame $O_{a}$. The residual errors of lever arm after measurement can be neglected compared with the positioning errors of USBL. The observation vectors and reference vectors are listed as follows

$$
\left\{\begin{array}{c}
\boldsymbol{m}=\boldsymbol{r}^{a} \\
\boldsymbol{n}=\boldsymbol{r}^{b}-\boldsymbol{L}^{b}
\end{array}\right.
$$

The accuracy of reference vector is relatively high, so the accuracy of attitude determination depends mainly on the accuracy of observation vector.

\section{B. Attitude determination method}

After constructing the observation vectors and reference vectors, the calibration of the installation error can be accomplished by the attitude determination algorithm. There are two important branches of attitude determination methods. The first one is concerned with the determination of the optimal matrix directly, while the other is to solve the corresponding optimal quaternion [24]. A well-known attractive feature of the quaternion is that the formulation of the attitude dynamics in terms of the quaternion is linear and nonsingular [23]. The attitude determination method used in this paper, named optimization-based quaternion method [25], can calculate the quaternion corresponding to the installation error angle. The principle of attitude determination method is described as follows.

The vector observation-based measurement model for installation error angle is expressed in quaternion form as follows:

$$
\boldsymbol{q}_{b}^{a} \otimes \boldsymbol{n} \otimes\left(\boldsymbol{q}_{b}^{a}\right)^{*}=\mathbf{m}
$$

Where the $\boldsymbol{q}_{b}^{a}$ is the attitude quaternion corresponding to the attitude matrix $\boldsymbol{C}_{b}^{a}$. The quaternion $\left(\boldsymbol{q}_{b}^{a}\right)^{*}$ indicates the conjugate quaternion of the corresponding quaternion $\boldsymbol{q}_{b}^{a}$. Operator $\otimes$ represents quaternion multiplication.

Multiplying both sides of (19) by $\boldsymbol{q}_{b}^{a}$, the following equation can be obtained.

$$
\boldsymbol{q}_{b}^{a} \otimes \boldsymbol{n}=\mathbf{m} \otimes \boldsymbol{q}_{b}^{a}
$$

The following two matrixes are constructed according the (20).

$$
\begin{aligned}
& \boldsymbol{M}(\boldsymbol{n})=\left[\begin{array}{cc}
0 & -\boldsymbol{n}^{T} \\
\boldsymbol{n} & -(\boldsymbol{n} \times)
\end{array}\right] \\
& \boldsymbol{M}(\boldsymbol{m})=\left[\begin{array}{cc}
0 & -\boldsymbol{m}^{T} \\
\boldsymbol{m} & (\boldsymbol{m} \times)
\end{array}\right]
\end{aligned}
$$

After constructing the matrix $M(\boldsymbol{n})$, the left side of (20) can be transformed to:

$$
\boldsymbol{q}_{b}^{a} \otimes \boldsymbol{n}=\boldsymbol{M}(\boldsymbol{n}) \boldsymbol{q}_{b}^{a}
$$

In the same way, the right side of (20) can be transformed to:

$$
\mathbf{m} \otimes \boldsymbol{q}_{b}^{a}=\boldsymbol{M}(\boldsymbol{m}) \boldsymbol{q}_{b}^{a}
$$

The measurement model can be rewritten as

$$
[\boldsymbol{M}(\boldsymbol{m})-\boldsymbol{M}(\boldsymbol{n})] \boldsymbol{q}_{b}^{a}=\mathbf{0}
$$

According to the optimization-based quaternion method [25], the above attitude determination problem can be transformed into the following optimal solution problem.

$$
\min _{q} \int_{t 0}^{t}\left\|[\boldsymbol{M}(\boldsymbol{m})-\boldsymbol{M}(\boldsymbol{n})] \boldsymbol{q}_{b}^{a}\right\| d t=\min _{q}\left(\boldsymbol{q}_{b}^{a}\right)^{T} \boldsymbol{K}\left(\boldsymbol{q}_{b}^{a}\right)
$$

Where, $\quad \boldsymbol{K}=\int_{t 0}^{t}\left([\boldsymbol{M}(\boldsymbol{m})-\boldsymbol{M}(\boldsymbol{n})]^{T}[\boldsymbol{M}(\boldsymbol{m})-\boldsymbol{M}(\boldsymbol{n})]\right) d t$. The constraint condition of quaternion $\boldsymbol{q}_{b}^{a}$ is $\left(\boldsymbol{q}_{b}^{a}\right)^{T}\left(\boldsymbol{q}_{b}^{a}\right)=1$. The eigenvector of the matrix K corresponding to its minimum eigenvalue is the sought attitude quaternion $\boldsymbol{q}_{b}^{a}$.

For more clarity, the structure of the calibration method of installation error for USBL based on attitude determination is as shown in Fig 4.

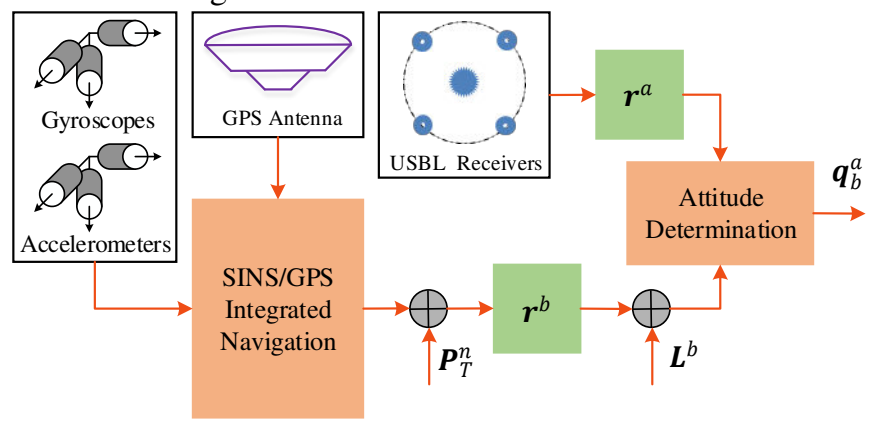

Fig. 4. The structure of the proposed calibration method.

\section{Simulation}

The performance of the calibration method is assessed in the simulation to verify the effectiveness of the proposed method. The route of the simulation tests in this paper is shown in Fig 5. In the simulation experiment, the transponder is located at $[-300 \mathrm{~m} ; 600 \mathrm{~m} ;-500 \mathrm{~m}]$ relative to the starting point. The origin of b-frame coincides with that of a-frame in the simulation 
experiment, so there is no displacement between SINS and USBL. The parameters of the sensors are set as Tab I, which includes the inertial sensors, USBL system and GPS system. The simulation test lasts 900 s.

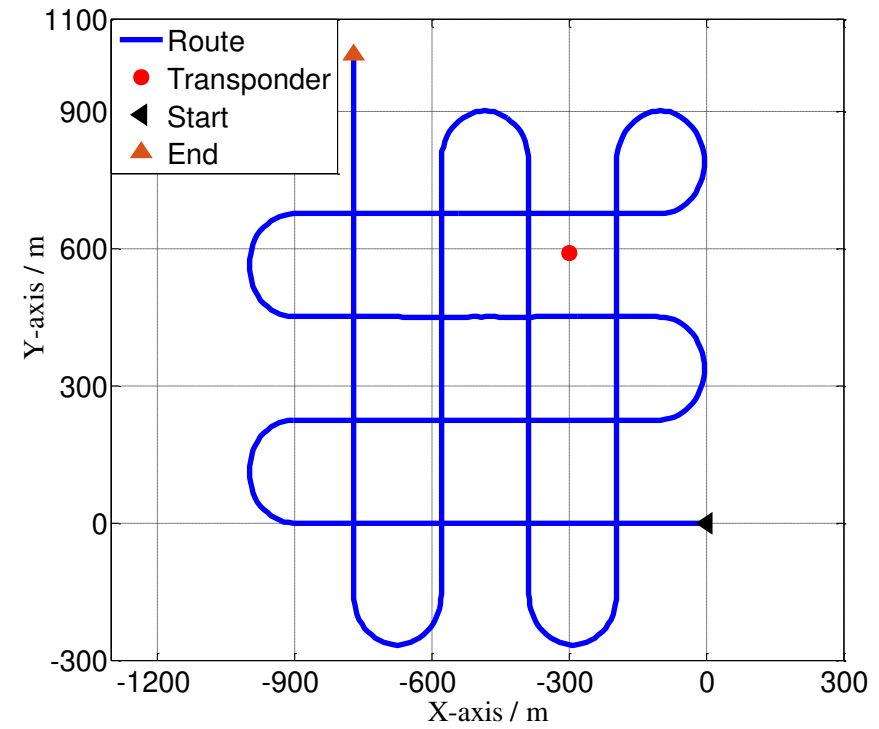

Fig. 5. Trajectory of the simulation experiment. TABLE I

INSTRUMENT PARAMETERS IN THE SIMULATION EXPERIMENT

\begin{tabular}{ccc}
\hline \hline & Parameters & Value \\
\hline \multirow{2}{*}{ USBL } & Positioning error & $1 \%$ slant range \\
& Direction error & $0.2^{\circ}$ \\
& Depth error & $1 \mathrm{~m}$ \\
\hline \multirow{3}{*}{ SINS } & Gyros biases & $0.01 \% \mathrm{~h}$ \\
& Gyros random error & $0.01 \% / \sqrt{\mathrm{h}}$ \\
& Accelerometers biases & $100 \mu \mathrm{g}$ \\
& Accelerometers random error & $100 \mu \mathrm{g} / \sqrt{\mathrm{Hz}}$ \\
\hline RTK & Position error & $0.05 \mathrm{~m}$ \\
\hline \multirow{2}{*}{ SINS/RTK } & Horizontal angle error & $0.05^{\circ}$ \\
& Heading angle error & $0.1^{\circ}$ \\
\hline \hline
\end{tabular}

The original data of the USBL system corresponding to the simulation route are shown in Fig 6, including two directions $\alpha_{x}$ and $\alpha_{y}$, and slant range $\mathrm{R}$.
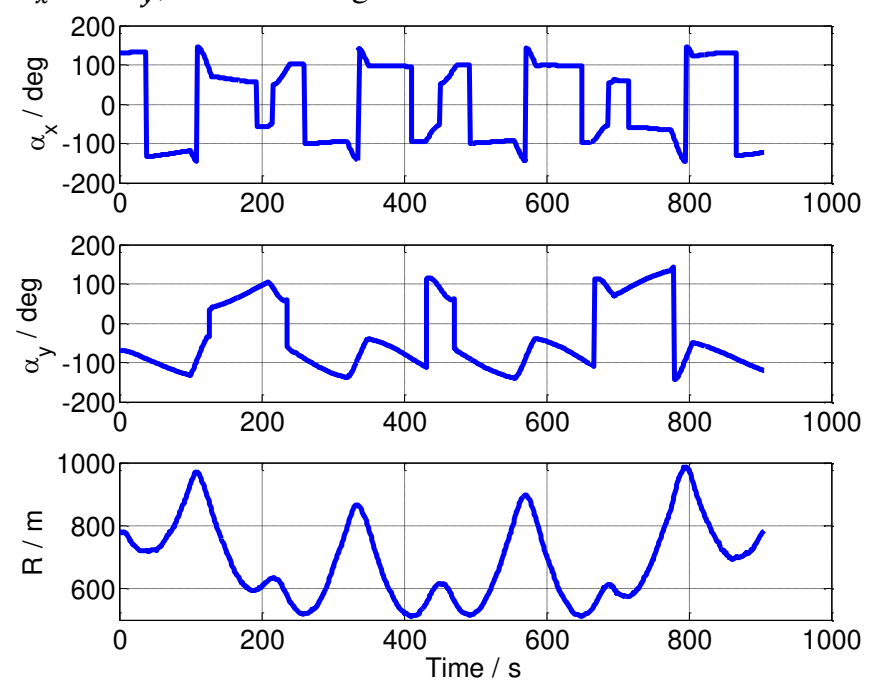

Fig. 6. Directions and slant range of USBL system in simulation experiment.

In this simulation experiment, the installation error angles between SINS and USBL are set to $\left[1.6^{\circ},-2.3^{\circ}, 4.5^{\circ}\right]$. The main purpose of this experiment is to verify the performance of the calibration method and to qualitatively analyze the USBL positioning results after the compensation of the installation error. The error curves of the installation error angles estimated by the proposed calibration method are shown in the Fig 7. As can be seen from Fig 7, the estimation errors of the three angles converge to less than $0.1^{\circ}$ after $200 \mathrm{~s}$.
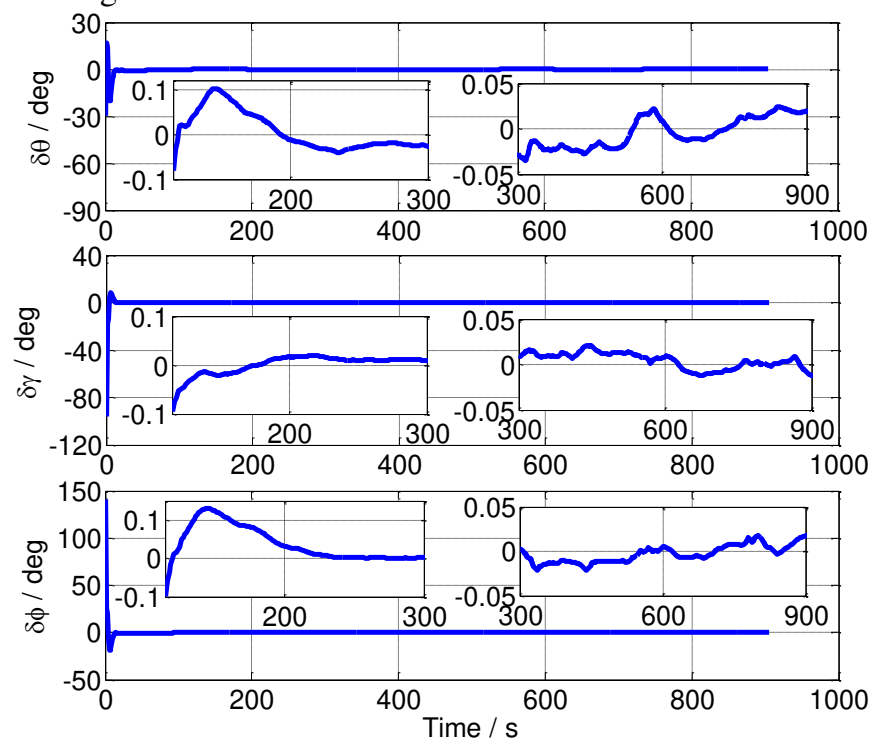

Fig. 7. Error curves of the installation error angles estimated by the proposed calibration method in simulation experiment.

Tab II gives the statistical results of the errors estimated by the proposed calibration method in detail, including mean (MN) error, standard deviation (SD) and root mean square (RMS) error. As can be seen from the Tab II, RMS error is less than 0.025 degrees after $300 \mathrm{~s}$.

TABLE II

STATISTICAL RESULTS OF CALIBRATION ERRORS IN SIMULATION EXPERIMENT

\begin{tabular}{ccccc}
\hline \hline \multicolumn{2}{c}{ Angle error } & $10-300 \mathrm{~s}\left(^{\circ}\right)$ & $301-600 \mathrm{~s}\left(^{\circ}\right)$ & $601-900 \mathrm{~s}\left(^{\circ}\right)$ \\
\hline \multirow{2}{*}{$\mathrm{MN}$} & Pitch & -0.2014 & -0.0137 & 0.0052 \\
& Roll & 0.0113 & 0.0114 & -0.0024 \\
& Yaw & -0.2095 & -0.0096 & 0.0035 \\
\hline \multirow{2}{*}{ SD } & Pitch & 0.5150 & 0.0153 & 0.0124 \\
& Roll & 0.2276 & 0.0038 & 0.0061 \\
& Yaw & 0.5549 & 0.0064 & 0.0074 \\
\hline \multirow{2}{*}{ RMS } & Pitch & 0.5521 & 0.0205 & 0.0134 \\
& Roll & 0.2275 & 0.0120 & 0.0065 \\
& Yaw & 0.5922 & 0.0116 & 0.0082 \\
\hline \hline
\end{tabular}

In order to verify the performance of the algorithm more intuitively, this method is compared with the least square method [16] and matrix decomposition method [14]. Tab III shows the mean error comparison results of several calibration methods. It can be seen from Tab III that the performance of the calibration method proposed in this paper is better than that of the other two methods.

TABLE III

THE MEAN ERROR RESULTS OF SEVERAL CALIBRATION ALGORITHMS

\begin{tabular}{|c|c|c|c|}
\hline Methods & $\begin{array}{c}\text { Pitch } \\
\text { Error }\left(^{\circ}\right)\end{array}$ & $\begin{array}{c}\text { Roll } \\
\text { Error }\left(^{\circ}\right)\end{array}$ & $\begin{array}{c}\text { Yaw } \\
\text { Error }\left(^{\circ}\right)\end{array}$ \\
\hline Least Square Method & 0.0141 & 0.0667 & 0.1033 \\
\hline $\begin{array}{l}\text { Matrix Decomposition } \\
\text { Method }\end{array}$ & 0.0285 & 0.0123 & 0.0599 \\
\hline The Proposed Method & 0.0052 & -0.0024 & 0.0035 \\
\hline
\end{tabular}



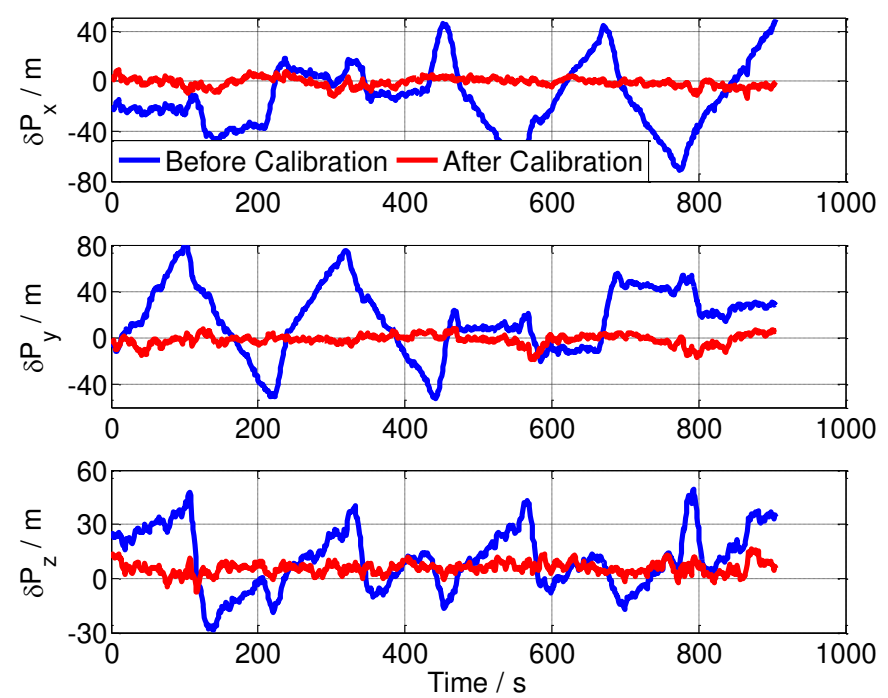

Fig. 8. Comparison of USBL positioning errors with or without compensation for installation error angle.

Fig 8 shows the positioning error curves of USBL position system. The red curve in Fig 8 is the positioning error curve after compensating the installation error angle, while the blue curve is the positioning error curve without compensating the installation error angle. It can be seen from Fig 8 that there is fluctuation in the blue curve. The causes of this phenomenon are analyzed as follows. On the one hand, due to the existence of installation error angle, the direction measured by USBL system contains error, namely $\widetilde{\alpha}=\alpha+\Delta \alpha$. Where $\widetilde{\alpha}$ indicates the direction obtained from the measurement of USBL system, $\alpha$ represents the true value of direction, and $\Delta \alpha$ represents the direction measurement error of USBL system caused by the installation error angle. On the other hand, the distance measurement results of USBL system are not affected by the installation error angle. The $\mathrm{x}$-axis position calculated by USBL system is $\mathrm{R} \cos \widetilde{\alpha}_{x}=R \cos \left(\alpha_{x}+\Delta \alpha_{x}\right)=$ $R \cos \alpha_{x} \cos \Delta \alpha_{x}-R \sin \alpha_{x} \sin \Delta \alpha_{x} \approx R \cos \alpha_{x} \cos \Delta \alpha_{x}$. It should be noted that since the installation error set in the simulation experiment is within $5^{\circ}$, the resulting USBL angle measurement error $\Delta \alpha_{x}$ is also small angle. Hence, the following formula is established, $\sin \Delta \alpha_{x} \ll \cos \Delta \alpha_{x}$. The $\mathrm{x}$-axis position error is calculated as $\Delta \mathrm{P}_{x} \approx R \cos \alpha(1-$ $\cos \Delta \alpha)$. Therefore, the error curve in the USBL positioning result without compensation for installation error generally changes with the fluctuation of the slant range in the simulation test.

From the comparison of positioning errors before and after installation error compensation as shown in Fig 8, we can qualitatively see the importance of installation error angle to positioning accuracy. Based on the above analysis, it can be concluded that the simulation experiment fully proves the correctness of the proposed calibration method.

\section{FIELD TEST}

In order to further verify the performance of the proposed method in the actual environment, a field test of calibration experiment was carried out at Yangtze River in Nanjing city, China. The USBL equipment in this field test was provided by the Hi-Target company. Several other calibration methods are used in the experiment to compare with the method proposed in this paper. They are as follows: (1) the calibration method based on matrix decomposition [14] is abbreviated as Scheme1; (2) the calibration method based on least square method [16] is marked as Scheme2; (3) the calibration method of the Hi-Target company is recorded as Scheme3; (4) the calibration method based on attitude determination proposed in this paper is recorded as Scheme4.

Next, the experimental equipment used in this field test is introduced, including SINS, USBL system, GPS and processing computer. The USBL system used in this experiment is the UB3000 of the Hi-Target company. The specific performance parameters of the product UB3000 are shown in the Tab IV. This product UB3000 mainly consists of two parts: transponders placed underwater, transducers and acoustic arrays installed on the bottom of the ship. The receiving array of the UB3000 uses four hydrophones and is placed in a square shape. The graphs of transponder and acoustic arrays are shown as Fig 9.

TABLE IV

PERFORMANCE PARAMETERS OF UB3000

\begin{tabular}{cc}
\hline \hline Parameters & Value \\
\hline Range & $3000 \mathrm{~m}$ \\
Positioning accuracy & $0.5 \mathrm{~m}+1 \% \mathrm{R}$ \\
Depth range of transponder & $2000 \mathrm{~m}$ \\
Update cycle & $3 \mathrm{~s}$ \\
\hline
\end{tabular}

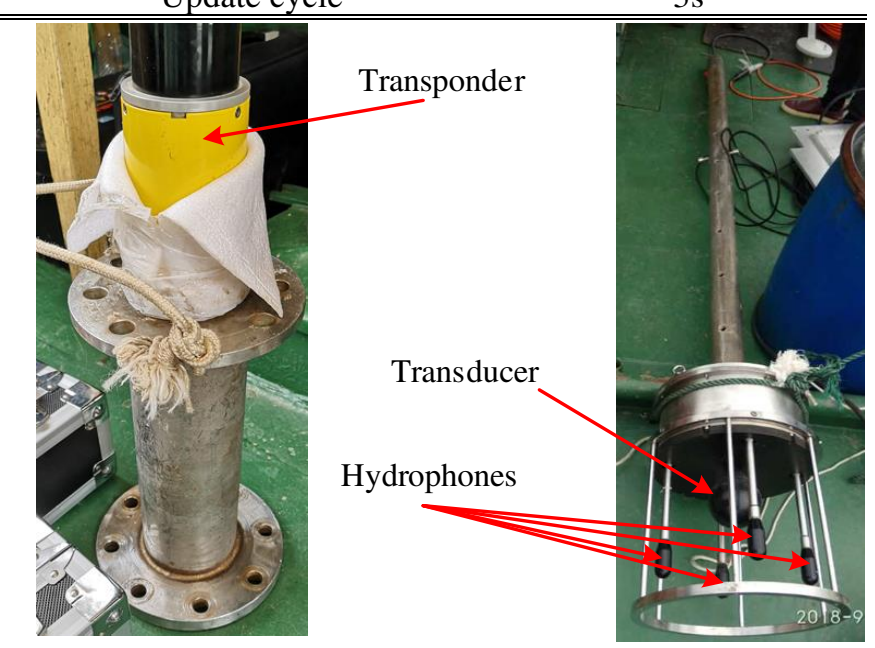

Fig. 9. Graphs of transponder and acoustic arrays in field test.

The attitude sensor and GPS used in the calibration experiment are shown in the Fig 10. The antenna 2 is a GPS receiving antenna, which is installed directly above the acoustic array. The RTK positioning of GPS with ambiguity integer solution is utilized in the field test, which has two functions. On the one hand, RTK is used to verify the positioning accuracy of USBL as the true value of position. On the other hand, it is used to correct the attitude error of SINS. The attitude sensor provides horizontal attitude information for USBL System. The heading angle information of USBL system is provided by dual antenna system consisting of antenna 1 and antenna 3 . 


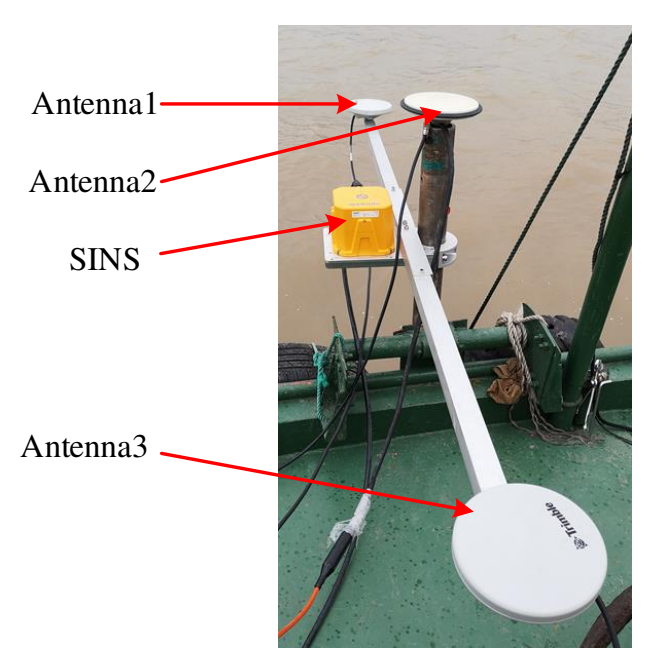

Fig. 10. Graphs of attitude sensor and GPS in field test.

The position of the transponder placed on the bottom of the river is shown by the yellow mark in the Fig 11. After the transponder is placed, the experimental ship moves a circular track around the transponder. The route is shown as the red curve in Fig 11. The original data of USBL corresponding to this route is shown in Fig 12, which contains two directions $\alpha_{x}$ and $\alpha_{y}$, and slant range R. As can be seen from the Fig 12, the slant range between the acoustic array and the transponder varies from $40 \mathrm{~m}$ to $90 \mathrm{~m}$.

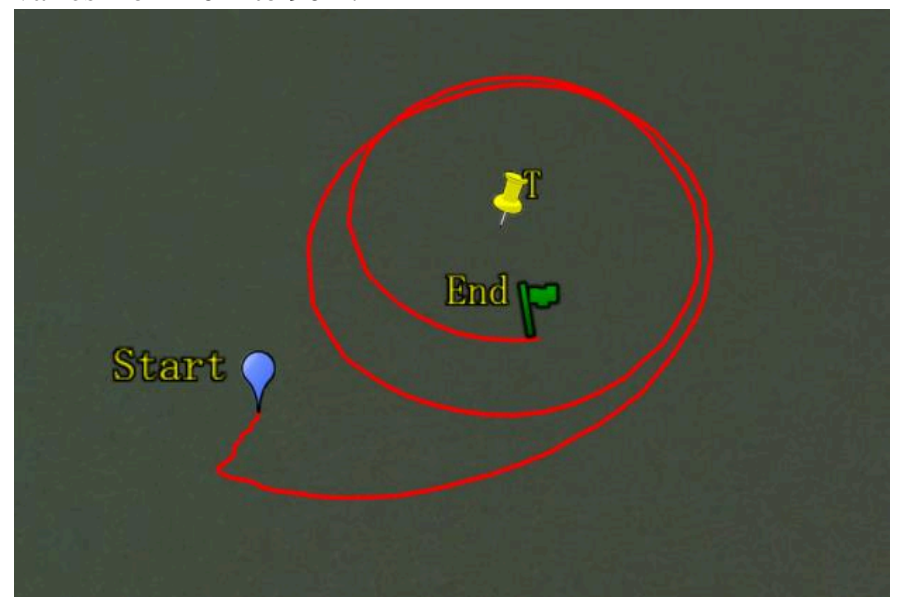

Fig. 11. Trajectory of the field test.
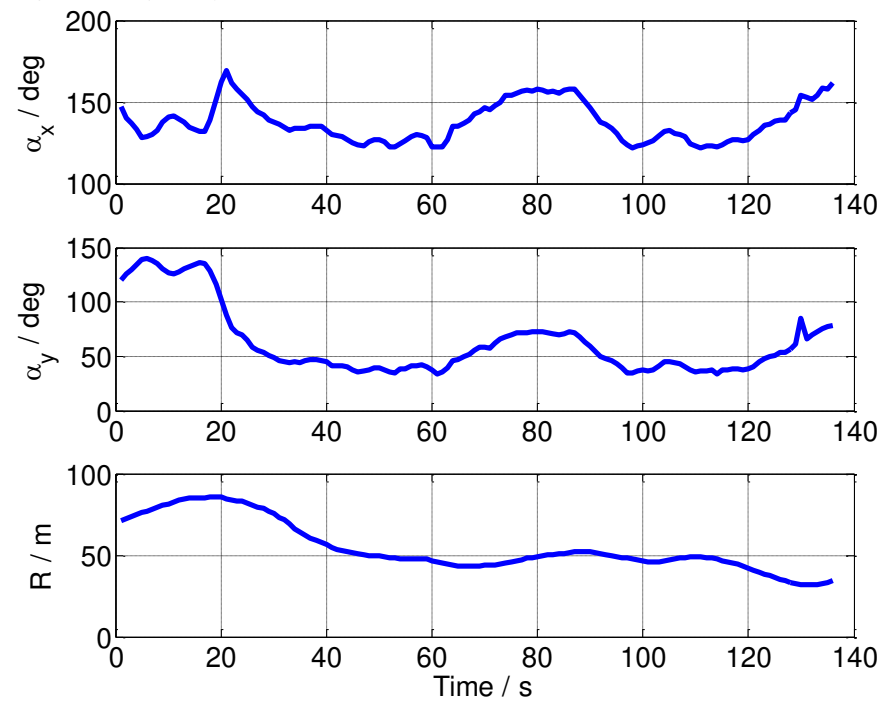

Fig. 12. Directions and slant range of USBL system in field test.

The displacement between the b-frame and the a-frame $\boldsymbol{L}^{b}$ are measured in advance, and the residual errors are neglected. The position of transponder is calibrated by the LBL positioning system. The observation vector $\boldsymbol{r}^{a}$ of the calibration method is calculated from the USBL raw data. The position of the transponder in b-frame $\boldsymbol{r}^{b}$ is calculated according to transponder position, GPS position and SINS attitude. The reference vector of the calibration method is obtained by compensating the arm $\boldsymbol{L}^{b}$ to the position vector $\boldsymbol{r}^{b}$. The installation error angle between USBL and SINS is solved using the calculated observation vector and reference vector. The result of the calibration method is as shown in the Fig 13. The $\theta, \gamma$ and $\phi$ of the $\mathrm{Y}$ axis in Fig 13 represent the three installation error angles, respectively. Tab $\mathrm{V}$ gives the calibration results of other methods.
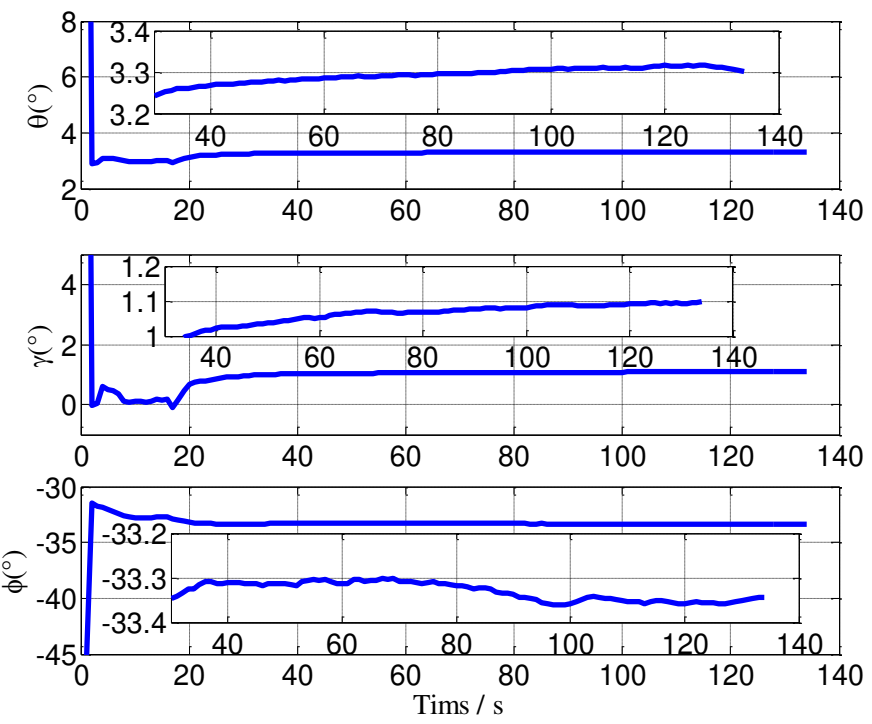

Fig. 13. Curves of the installation error angles estimated by the proposed calibration method in field test.

TABLE V

THE RESULT OF SEVERAL CALIBRATION METHODS IN FIELD TEST

\begin{tabular}{cccc}
\hline \hline Methods & $\theta\left(^{\mathbf{o}}\right)$ & $\gamma\left(\left(^{\mathbf{o}}\right)\right.$ & $\phi\left(^{\mathbf{o}}\right)$ \\
\hline Scheme1 & 4.4223 & -6.8863 & -34.3020 \\
Scheme2 & -3.2630 & -7.3991 & -34.9293 \\
Scheme3 & -0.9879 & 1.7492 & -34.5030 \\
Scheme4 & 3.1808 & 1.0906 & -33.3746 \\
\hline \hline
\end{tabular}

It can be seen from the Fig 13 that the fluctuation range of the three installation error angles estimated by the proposed method is stable within 0.1 degrees after 40 s. According to the calibration results of several methods given in the Tab $\mathrm{V}$, the angle $\phi$ of these methods is close, but the angles of $\theta$ and $\gamma$ are quite different. In the field test, the true installation error angle is unknown. The performance of several calibration methods cannot be evaluated directly from Tab V. We will verify the performance of several algorithms by comparing the positioning error of USBL system after installation error compensation.

Next, the effect of several calibration results is verified by comparing the positioning accuracy of USBL hydrophone after compensating the installation error angles. The transponder position calculated by LBL is set as known, and the hydrophone 
position is calculated by USBL system after compensating the installation error angle. The hydrophone position obtained by GPS is taken as the true value, then the position error of the hydrophone is calculated by the following equation. The error curves of the hydrophone position of the several methods are shown as Fig 14. The error statistics of several methods are given in Tab VI.

$$
\delta \boldsymbol{P}_{A}^{n}=\boldsymbol{P}_{A}^{n}-\boldsymbol{P}_{A_{G P S}}^{n}=\boldsymbol{P}_{T}^{n}-\boldsymbol{C}_{b}^{n} \boldsymbol{C}_{a}^{b} \boldsymbol{r}^{a}-\boldsymbol{P}_{A_{G P S}}^{n}
$$

Where the position $\boldsymbol{P}_{A_{G P S}}^{n}$ is the hydrophone position obtained by GPS.
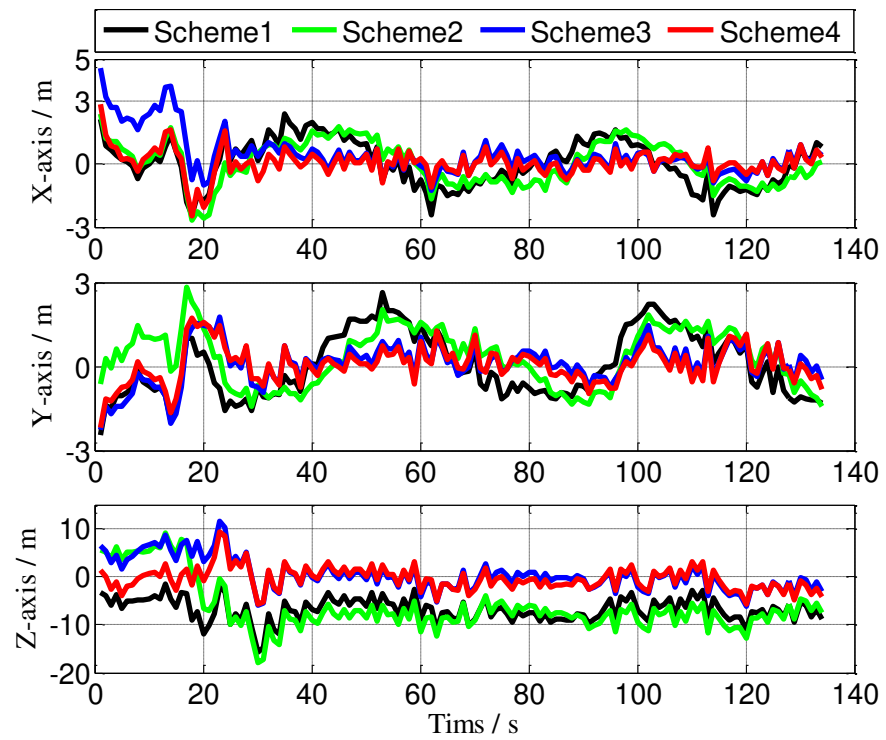

Fig. 14. Comparisons of USBL positioning errors of several calibration methods after compensating installation error angles. TABLE VI

THE POSITIONING RESULT OF SEVERAL CALIBRATION METHODS IN FIELD TEST

\begin{tabular}{|c|c|c|c|c|}
\hline Erro & tatistics & X-axis & Y-axis & Z-axis \\
\hline \multirow{4}{*}{$\mathrm{RMS} / \mathrm{m}$} & Scheme4 & 0.6456 & 0.6601 & 2.4532 \\
\hline & Scheme3 & 1.0988 & 0.7212 & 3.3245 \\
\hline & Scheme2 & 1.0498 & 1.0512 & 8.2196 \\
\hline & Scheme1 & 1.0974 & 1.13878 & 7.2388 \\
\hline \multirow{4}{*}{$\mathrm{SD} / \mathrm{m}$} & Scheme4 & 0.6450 & 0.6591 & 2.4264 \\
\hline & Scheme3 & 0.9986 & 0.7180 & 3.2946 \\
\hline & Scheme2 & 1.0524 & 0.9977 & 5.3241 \\
\hline & Scheme1 & 1.1005 & 1.1423 & 2.4324 \\
\hline \multirow{4}{*}{$\mathrm{MN} / \mathrm{m}$} & Scheme4 & 0.0628 & 0.0680 & -0.4187 \\
\hline & Scheme3 & 0.4666 & 0.0927 & 0.5290 \\
\hline & Scheme2 & 0.0547 & 0.3422 & -6.2793 \\
\hline & Scheme1 & 0.0487 & 0.0433 & -6.8212 \\
\hline
\end{tabular}

From the error curve given in Fig 14, it can be clearly seen that the error fluctuation range of the red curve representing the Scheme 4 is the smallest among four methods. Therefore, the installation error angle estimated by the proposed method is the closest to the true value. On the other hand, it can be seen from the Tab VI that the positioning errors of the proposed method are mostly less than those of the other three methods.

Next, the performances of several calibration methods are verified by comparing the position errors of transponders after compensating the installation error angles. The hydrophone position obtained by GPS is set as known, and the transponder position is calculated by USBL system after compensating the installation error angle. The transponder position calculated by LBL is taken as the true value, then the position error of the transponder is calculated by the following equation. The performance of calibration methods is reflected by analyzing the distribution of transponder position errors. Fig 15 gives the error distributions of the transponders position after compensating each calibration results.

$$
\delta \boldsymbol{P}_{T}^{n}=\boldsymbol{P}_{T_{U S B L}}^{n}-\boldsymbol{P}_{T}^{n}=\boldsymbol{P}_{A_{G P S}}^{n}+\boldsymbol{C}_{b}^{n} \boldsymbol{C}_{a}^{b} \boldsymbol{r}^{a}-\boldsymbol{P}_{T}^{n}
$$

Where the position $\boldsymbol{P}_{T_{U S B L}}^{n}$ is the transponder position in $\mathrm{n}$-frame obtained by USBL system after compensating the calibrated installation error.
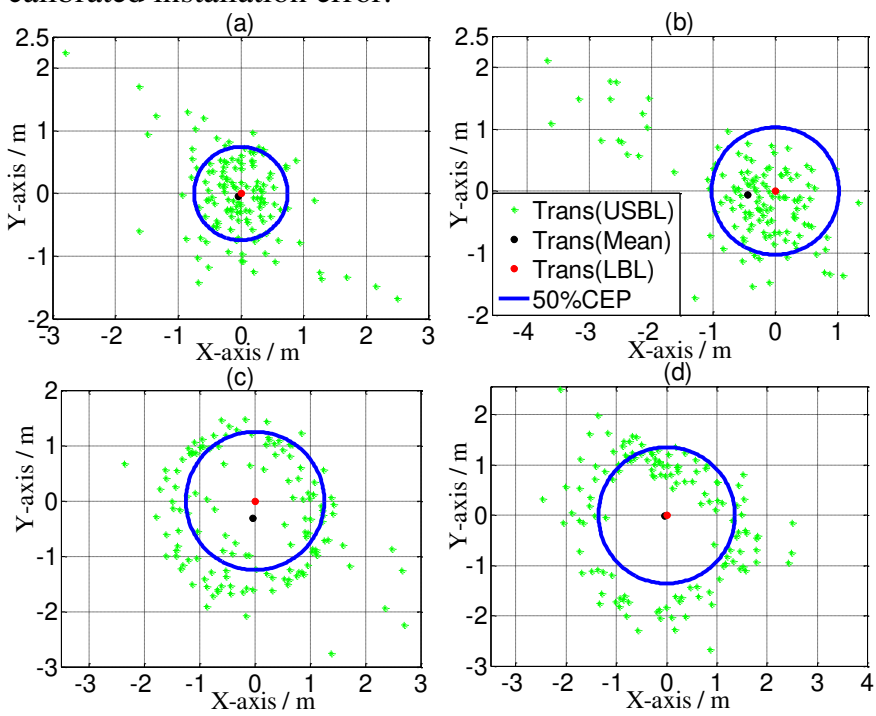

Fig. 15. The error distributions of the transponders after compensating calibration results.

(a) the result of scheme4; (b) the result of scheme3; (c) the result of scheme2; (d) the result of scheme1.

The scattered green dots in the Fig 15 represent the location values of the transponder obtained by the USBL position system in every update cycles. The black dot denotes the average position of the transponders obtained by USBL. The red dot represents the transponder position obtained by the LBL, which is treated as the true value of the transponder position. The blue circle indicates a 50\% circular error probable (CEP) of the transponder position obtained by USBL, while the position calculated by LBL is located as the center of the circle. As can be seen from the Fig 15, the position distribution of the transponders compensated by the proposed calibration method in this paper is most concentrated. And the 50\% CEP of the proposed method is the smallest among the four methods. The error statistics of the several methods are given in Tab VII-VIII. TABLE VII

THE 50\% CEP RESULT OF SEVERAL CALIBRATION METHODS

\begin{tabular}{cccc}
\hline \hline \multicolumn{2}{c}{ Error statistics } & $2 \mathrm{D} / \mathrm{m}$ & $3 \mathrm{D} / \mathrm{m}$ \\
\hline \multirow{4}{*}{$50 \%$ CEP } & Scheme4 & 0.5905 & 2.0705 \\
& Scheme3 & 0.6865 & 2.6437 \\
& Scheme2 & 1.4262 & 6.1908 \\
& Scheme1 & 1.5741 & 4.6674 \\
\hline \hline \multicolumn{4}{c}{ TABLE VIII }
\end{tabular}


THE STATISTICAL RESULT OF POSITIONING ERRORS OF SEVERAL CALIBRATION METHODS

\begin{tabular}{|c|c|c|c|c|}
\hline \multicolumn{2}{|c|}{ Error statistics } & $\mathrm{X}$-axis & Y-axis & Z-axis \\
\hline \multirow{4}{*}{$\mathrm{RMS} / \mathrm{m}$} & Scheme4 & 0.6514 & 0.6624 & 3.1058 \\
\hline & Scheme3 & 1.1044 & 0.7293 & 4.3783 \\
\hline & Scheme2 & 1.0691 & 1.05756 & 6.5898 \\
\hline & Scheme1 & 1.1351 & 1.1791 & 5.0693 \\
\hline \multirow{4}{*}{$\mathrm{SD} / \mathrm{m}$} & Scheme4 & 0.6519 & 0.6633 & 2.4265 \\
\hline & Scheme 3 & 1.011 & 0.7286 & 3.2946 \\
\hline & Scheme2 & 1.0723 & 1.0117 & 5.3241 \\
\hline & Scheme1 & 1.1388 & 1.1834 & 2.4326 \\
\hline \multirow{4}{*}{$\mathrm{MN} / \mathrm{m}$} & Scheme4 & -0.0498 & -0.0460 & -1.9500 \\
\hline & Scheme3 & -0.4537 & -0.0707 & -2.8977 \\
\hline & Scheme2 & -0.0417 & -0.3202 & 3.9106 \\
\hline & Scheme1 & -0.0357 & -0.0213 & 4.4525 \\
\hline
\end{tabular}

Tab VII gives the 50\% CEP errors of transponders at horizontal and three-dimensional positions. The root mean square error, standard deviation and mean error of the transponder errors corresponding to the four methods are given in Tab VIII. As can be seen from Tab VII, the circular error probable of the proposed method, whether horizontal error or three-dimensional error, is the smallest of the four methods. From the standard deviation and root mean square of transponder positioning error given in Tab VIII, the accuracy of the proposed method is the highest.

The method has been verified by simulation experiments and field experiments. The method proposed in this paper can complete calibration of USBL installation error angle not only in straight path but also in circular route. Regardless of the qualitative or quantitative analysis of the positioning error, the calibration method proposed in this paper is feasible in practical environment and has higher accuracy.

\section{CONCLUSION}

In this paper, a calibration method of USBL installation error based on attitude determination is proposed to accurately estimate the installation error angle for USBL. The installation error angle of USBL and SINS is constant in application, so the calibration of installation error angle can be completed by attitude determination method. Firstly, the vector observation model based on the installation error angle matrix is established. The calibration method proposed in this paper can be obtained by constructing observation vectors and reference vectors. In order to correct the accumulated attitude errors of SINS, the SINS/GPS integrated navigation method is used to obtain more accurate attitude results. The position of transponder needs to be calculated by LBL system in advance. The simulation experiment and field test are carried out, to verify the performance of the calibration method proposed in this paper. The results of simulation and field experiments show that the performance of the proposed calibration method is the best among several calibration methods. More importantly, the proposed method can complete the real-time calibration technology of installation error angle, and has no specific requirement for calibration experiment route. Based on the experiment and analysis of this paper, we can draw a conclusion that the proposed method has high application value in practical USBL calibration.

\section{REFERENCES}

[1] Y. X. Wang, T. Gao, Y. J. Pang, Y. G. Tang, "Investigation and optimization of appendage influence on the hydrodynamic performance of AUVs," Journal of Marine Science and Technology, 2019, 24(1): 297-305.

[2] L. J. Zhong, D. J. Li, M. W. Lin, R. Lin, C. J. Yang, "A Fast Binocular Localization Method for AUV Docking," Sensors, 2019, 19(7), 1735.

[3] G. D. Xiao, B. Wang, Z. H. Deng, M. Y. Fu, Y. Ling, et al. "An acoustic communication time delays compensation approach for master-slave AUV cooperative navigation," IEEE Sensors Journal, 2017, 17(2): 504-513.

[4] T. Alexandri, R. Diamant. "A Reverse Bearings Only Target Motion Analysis for Autonomous Underwater Vehicle Navigation,". IEEE Transactions on Mobile Computing, 2019, 18(3): 494-506.

[5] P. J. Liu, B. Wang,Z. H. Deng, M. Y. Fu, "INS/DVL/PS tightly coupled underwater navigation method with limited DVL measurements," IEEE Sensors Journal, 2018, 18(7): 2994-3002.

[6] B. N. Jin, X. S. Xu, T. Zhang, "Robust time-difference-of-arrival (TDOA) localization using weighted least squares with cone tangent plane constraint," Sensors, 2018, 18(3): 778.

[7] T. W. Zhang, X. X. Wang, J. L. Tang, S. J. Qin, "Technical Status and Development Trend of Long Range USBL," Ship Electronic Engineering, 2018, 38(10): 1-6.

[8] J. Huang, S. G. Yan, "An Improvement of Long Baseline System Using Particle Swarm Optimization to Optimize Effective Sound Speed," Marine Geodesy, 2018, 41(5): 439-456.

[9] H. H. Chen. "The estimation of angular misalignments for ultra-short baseline navigation systems. Part I: numerical simulations," The Journal of Navigation, 2013, 66(4): 561-578.

[10] B. N. Jin, X. S. Xu, T. Zhang, X. J. Sun, "USBL technology and its applications in ocean engineering," Navigation Positioning \& Timeing, 2018, 5(4): 8-20.

[11] M. Morgado, P. Oliveira, C. Silvestre, J. F. Vasconcelos, "USBL/INS tightly-coupled integration technique for underwater vehicles," in Proceedings of International Conference on Information Fusion, Florence, Italy, Jul. 2006, pp. 1-8.

[12] M. Morgado, P. Oliveira, C. Silvestre, "Tightly coupled ultrashort baseline and inertial navigation system for underwater vehicles: An experimental validation," Journal of Field Robotics, 2013, 30(1): $142-170$.

[13] B. Wang, J. Liang, Y. G. Wang, H. N. Weng, Q. Zhang, "SINS/USBL integrated navigation fault-tolerant method with chi-square test," in Proceedings of IEEE ICINS, Saint Petersburg, Russia, 2018: 1-4.

[14] D. J. Sun, J. Ding, C. E. Zheng, W. M. Huang, "Angular misalignment calibration method for ultra-short baseline positioning system based on matrix decomposition," IET Radar, Sonar \& Navigation, 2018, 13(3): 456-463.

[15] B. G. Yang, C. E. Zheng, D. L. Zhang, Z. Li, "Nonlinear parameter estimation for calibration and installation of an ultra-short baseline system," Journal of Harbin Engineering University, 2010, 31(7): 915-919.

[16] C. E. Zheng, D. J. Sun, D. L. Zhang, X. Li, "Research the calibration technology of the installation error in ultra-short baseline acoustic positioning system," Computer Engineering and Applications, 2007, 43(8): 171-173

[17] C. E. Zheng, D. J. Sun, D. L. Zhang, X. Li, "Precise calibration of installation error in ultra-short baseline acoustic positioning systems," Journal of Harbin Engineering University, 2007, 28(8): 894-898.

[18] X. Z. Zhang, Y. Zhang, "Research on the calibration method of the array installation error in ultra-short baseline underwater acoustic locating system," Mine Warfare \& Ship Self-Defense, 2012, 20(3): 28-31.

[19] M. Yu, "In-situ calibration of transceiver alignment for a high-precision USBL system," in Proceedings of IEEE ICCASM, Taiyuan, China, 2010, 11: V11-84-V11-87.

[20] J. W. Tong, X. S. Xu, T. Zhang, L. Zhang, Y. Li, "Study on Installation Error Analysis and Calibration of Acoustic Transceiver Array Based on SINS/USBL Integrated System," IEEE Access, 2018, 6: 66923-66939.

[21] D. Choukroun, H. Weiss, I. Y. Bar-Itzhack, Y. Oshman, "Quaternion estimation from vector observations using a matrix Kalman filter," IEEE Transactions on Aerospace and Electronic Systems, 2012, 48(4): $3133-3158$ 
[22] F. L. Markley, J. L. Crassidis, "Matrices, Vectors, Frames, Transforms" in Fundamentals of spacecraft attitude determination and control, $1^{\text {st }}$, New York, USA: Springer, 2014, pp. 17-65.

[23] F. L. Markley, J. L. Crassidis, "Static Attitude Determination Methods" in Fundamentals of spacecraft attitude determination and control, $1^{\text {st }}, \mathrm{New}$ York, USA: Springer, 2014, pp. 182-233.

[24] D. Choukroun, I. Y. Bar-Itzhack, Y. Oshman, "Optimal-REQUEST algorithm for attitude determination," Journal of Guidance, Control, and Dynamics, 2004, 27(3): 418-425.

[25] M. P. Wu, Y. X. Wu, X. P. Hu, D. W. Hu, "Optimization-based alignment for inertial navigation systems: Theory and algorithm," Aerospace Science and Technology, 2011, 15(1): 1-17.

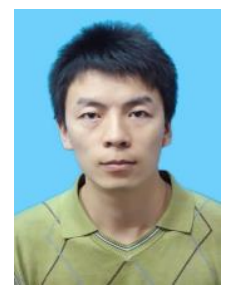

system.

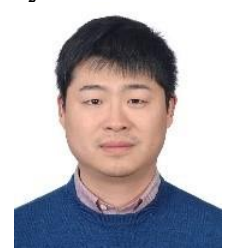

Yongyun Zhu was born in Xinxiang, Henan province, in 1991. He is currently pursuing the Ph.D. degree in navigation, guidance, and control at Southeast University, Nanjing, China. His current research interests include strap-down inertial navigation, integrate navigation, underwater acoustic navigation

Tao Zhang received the $\mathrm{Ph} . \mathrm{D}$. degree in navigation, guidance, and control from Southeast University, Nanjing, China, in 2008.

He is currently a professor with the School of Instrument Science and Engineering, Southeast University. His research interests include the ship's acoustic navigation system, LBL, USBL, initial alignment for inertial navigation, integrated navigation, and MEMS inertial technology.

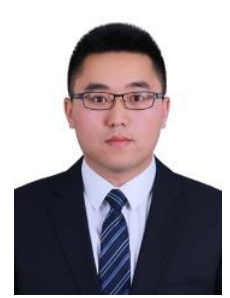

Shouquan $\mathrm{Xu}$ received master degree in computer science and technology from Nanjing University of Aeronautics and Astronautics, Nanjing, China, in 2014.

$\mathrm{He}$ is currently a software test engineer of Jiangsu Automation Research Institute. His research interests include software testing, software quality and reliability.

Hyo-Sang Shin received his BSc from Pusan National University in 2004 and gained an MSc on flight dynamics, guidance and control in Aerospace Engineering from KAIST and a $\mathrm{PhD}$ on cooperative missile guidance from Cranfield University in 2006 and 2010, respectively. He is currently a Professor on Guidance, Control and Navigation Systems in Autonomous and Intelligent Systems Group at Cranfield University. His current research interests include multiple target tracking, adaptive and sensor-based control, and distributed control of multiple agent systems.

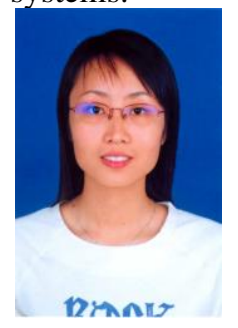

Peijuan Li was born in Yangzhong, Jiangsu, She was from Jiangsu, China. She received the B.S. degree in computer science and technology from Nanjing University of Information Science \& Technology, Nanjing, Jiangsu, in 2004. She received the M.S. degree in intelligent control and intelligent system from Jiangsu University of Science and Technology, Zhenjiang, Jiangsu, in 2007 and the Ph.D. degree in navigation guidance and control from Southeast University, Nanjing Jiangsu, in 2012. Her research interest includes inertial device test, matching algorithm of underwater terrain-aided navigation system and data fusion of autonomous underwater vehicle integrated navigation system.

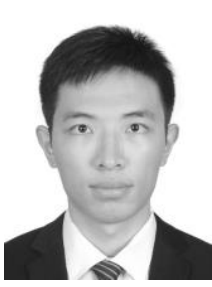

Bonan Jin was born in Nantong, Jiangsu province, in 1990. He is currently pursuing the Ph.D. degree in instrument science and technology with Southeast University, Nanjing, China. His current research interests include strap-down inertial navigation, hydro-acoustic positioning.

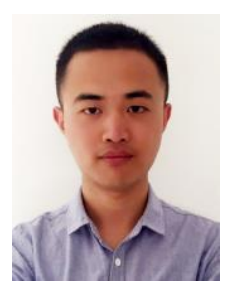

Liang Zhang was born in Yangzhou, Jiangsu province. He is currently pursuing the Ph.D. degree in instrument science and technology at Southeast University, Nanjing, China. His research interest includes inertial navigation and integrated navigation technology and calibration algorithm which applied in the field of underwater navigation technology.

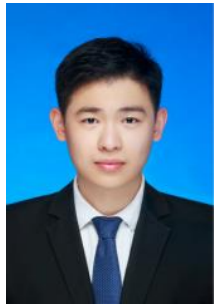

Chengcheng Weng was born in Fuzhou, Fujian province, in 1995. He received the B.S. degree in measurement and control technology and instrument from Southeast University, Nanjing, China, in 2017. He is currently pursuing the M.S. degree in Navigation, Guidance and Control with Southeast University, Nanjing, China. His research interests include strap-down inertial navigation and hydro-acoustic positioning.

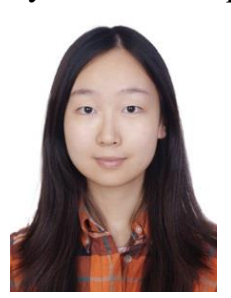

Yao Li received the Ph.D. degree in guidance navigation and control from Southeast University, Nanjing, China, in 2012. She studied at the University of New South Wales, Australia, in 2010.

She is currently a lecturer in the School of Instrument Science and Engineering, Southeast University. Her research interests include strapdown inertial navigation system (SINS), information fusion of integrated navigation system. 
2020-05-18

\title{
A calibration method of USBL
} installation error based on attitude determination

\author{
Zhu, Yongyun
}

IEEE

Zhu Y, Zhang T, Xu S, et al., (2020) A calibration method of USBL installation error based on attitude determination. IEEE Transactions on Vehicular Technology, Volume 69, Issue 8 , August 2020, pp. 8317-8328

https://doi.org/10.1109/TVT.2020.2995599

Downloaded from Cranfield Library Services E-Repository 